\title{
25 Research Suare \\ Gut Microbiome of Century-Old Snail Specimens Stable Across Time in Preservation
}

Bridget Chalifour ( $\square$ bridget.chalifour@colorado.edu )

University of Colorado Boulder

Jingchun Li

University of Colorado Boulder

Leanne Elder

University of Colorado Boulder

\section{Research Article}

Keywords: Gut Microbiome, Mollusk, Natural History Museum, Gastropod, 16S rRNA gene, Invertebrates

Posted Date: February 24th, 2022

DOI: https://doi.org/10.21203/rs.3.rs-1328491/v1

License: (1) This work is licensed under a Creative Commons Attribution 4.0 International License.

Read Full License 


\section{Abstract \\ Background}

Oreohelix strigosa (Rocky Mountainsnail) is a land snail found in the talus slopes of the Rocky Mountains. The University of Colorado's Museum of Natural History has densely sampled Oreohelix for the past century; many are preserved in ethanol and available for molecular research. While microbiome compositions in other systems are not affected by short-term field season preservation, the effects of decades-long preservation have yet to be assessed.

\section{Results}

We used 16S rRNA (ribosomal ribonucleic acid) gene amplicon sequencing to examine Oreohelix strigosa gut microbiomes from museum specimens across a 98-year range, as well as within short term preservation treatments collected in 2018. Treatment groups included samples extracted fresh, without preservation; samples starved prior to extraction; and samples preserved for 1 month, 6 months, and 9 months. General microbiome composition was similar across all years. Sample groups belonging to specific years, or specific short-term treatments, showed unique associations with select bacterial taxa. Collection year was not a significant predictor of microbial richness, though unpreserved short-term treatments showed significantly higher richness than preserved treatments. While year was a significant factor in microbiome composition, it did not explain much of the variation across samples. Other factors like location collected and drought index were also significant drivers of community composition and explained as much or more of the variability.

\section{Conclusions}

This study is the first to examine animal host associated microbiome change across a period of nearly one century. Generally, geographic location was a greater factor in shaping gut microbiome composition, rather than year collected. Consistent patterns across this temporal range indicate that historic specimens can answer many ecological questions surrounding the host associated microbiome.

\section{Introduction}

The microbiome is an evolutionary adaptation that has allowed many animals to capture and utilize resources in their environment [1]. The gut microbiome is particularly important to study as it provides insight into both host-specific and population level subtle changes in environmental patterns and microbial communities. It is imperative to understand how microbiomes shift over broad temporal and spatial gradients as changes in the microbiome can impact host response to stressors [2]. Analyzing past trends in microbiome shifts is also paramount to predicting future trends and safeguarding rare species that depend on their symbiotic microbes [3]. Historic museum specimens provide the long-term data 
needed to form a more comprehensive picture of how environmental changes impact microbial communities and predict how microbiomes will shift due to future changes [2,4]. The specimens also provide a unique and often underappreciated means of gathering ecological information spanning wide geographic ranges [5].

So far, studies using museum collections have mainly focused on the host, and not organisms living on or within the host [6], especially not microbiomes associated with the specimens. Microbiomes associated with other biological samples can now be routinely characterized, thanks to the development of high-throughput sequencing approaches, which are cost-effective and able to characterize previously difficult to culture bacteria [7]. This development has widened the possible scale and narrowed the effort needed to describe the microbiomes of museum specimens, allowing microbiome researchers to conduct expansive studies with limited field work.

Many museum specimens are preserved at room temperature in ethanol. To effectively use these samples as representatives of the past and conduct microbiome analysis, we must ensure deoxyribonucleic acid (DNA) degradation is minimal after long periods of storage in these conditions. Previous studies have compared the efficacy of various initial preservation and extraction methods in obtaining high concentration DNA from vertebrate and invertebrate specimens $[8,9,10]$. Other studies have assessed methods of sequencing degraded DNA from historic specimens [11, 12]. Generally, while some Polymerase Chain Reaction (PCR) success is age-dependent, it is possible to obtain viable genetic information from host specimens up to hundreds of years old. In fact, fixation, storage, sample conservation and documentation of the specimens' history, rather than extraction methodology, are often the more crucial factors for successful use of historic DNA $[13,14,15]$.

While microbiome compositions in animal hosts are not significantly altered by short-term preservation [14], the effects of decades-long preservation require further study. As far as we know, no studies have directly tested how different time periods in ethanol preservative impact animal-associated microbial abundance, diversity, and community structure over a decades-long time scale. To address this issue, we need a model species with an extensive presence and wide temporal range in museum collections and known to harbor a core gut microbiome.

Land snails are dispersal-limited, due to the high energy cost of movement [16]. As low-vagility organisms, many land snails must survive within a limited range. If a habitat does not meet certain qualifications, they must adapt or perish. The gastropod microbiome is theorized to influence the host's physiological state, confer protection against pathogens, and regulate host immune function, along with enabling host dietary diversity $[17,18]$. Therefore, the snail microbiome may play an important role in the host's adaptation, tolerance to environmental perturbations, and immune system functioning [7, 19]. Oreohelix strigosa, more commonly known as the Rocky Mountainsnail, is a pulmonate terrestrial gastropod belonging to the class Gastropoda (Gould, 1846, Fig. 1A, B). O. strigosa is widespread across the mountainous western United States, found in a multitude of habitats, ranging from grassy fields to the talus slopes of the Rocky Mountains. More importantly, the University of Colorado Museum of Natural 
History (CUMNH) has densely sampled 0 . strigosa for the past century, many are preserved in ethanol and therefore appropriate for molecular research. The CUMNH wet collection contains thousands of preserved specimens of $O$. strigosa, ranging in collection date from 1915 to 2019. Previous research has indicated the presence of a stable gut microbiome in 0 . strigosa specimens [20], making it an ideal system to compare microbiome quality through time.

The aims of this study were threefold: (1) to test the applicability of high-throughput sequencing techniques on describing animal gut microbiomes using museum specimens (2) to compare historic museum specimen microbiomes to contemporary samples and (3) to explore potential driving factors of microbiome variability in museum samples. We investigated the impacts of preservation in both longterm (15-98 years) and short-term (0-9 months) ethanol-preserved samples. We examined taxonomic changes, changes in microbial composition, as well as changes in microbial richness, or number of operational taxonomic units (OTUs) within each individual's gut microbiome.

Though there is a substantial dearth of research examining the long-term effects of museum preservation on the microbiome, one study has shown that museum-preserved fish microbiomes can be captured through high-throughput sequencing, though this study had small sample sizes likely due to formalinfixated samples which contributed to high dropout rates in the data [6]. Another study by Neu et al. (2021) showed that bivalve tissue preserved in ethanol for up to ten years was successful at providing highresolution information regarding trends in microbial community compositions [2]. Other studies have found that microbial richness is less dramatically affected by most preservation methodologies in shortterm storage experiments [21], and that methodological consistency is more important than time of preservation [22]. Based on these past findings, we predicted that microbial community composition and diversity would not significantly differ among sample populations from different years. Collectively, this work advances our knowledge of the applicability of decades-old museum specimens to high-throughput sequencing projects and broadens knowledge on terrestrial gastropod gut microbiomes.

\section{Materials And Methods}

This study consists of data from 112 snails from two sources: 55 historical collected specimens from the University of Colorado Museum of Natural History's (CUMNH) Invertebrate Zoology collections (for specimen information, see Supplementary Table 1), and 57 field collected specimens from localities within the state of Colorado (deposited in the CUMNH Invertebrate Zoology collections, see Supplementary Table 1).

\section{Museum Sampling}

The 55 historical specimens from the CUMNH collections were collected between 1920 and 2004. Sample selection from museum collections was based on 1) the sample size within each jar - in order to leave intact specimens for future study and morphological reference, we only sampled from lots that contained at least 15 specimens; 2) the distance between time points, as we strived to achieve both even spacing between years and a wide temporal spread of years in preservation. Based on these criteria, 3 samples 
were taken from the year 1920, 15 from 1974, 2 from 1980, 13 from 1982, 2 from 2000, and 20 from 2004 (Table 1).

Table 1. Metadata from all snail samples.

\begin{tabular}{|c|c|c|c|c|c|c|}
\hline Location: & $\begin{array}{l}\text { GPS Coordinates } \\
\text { (Estimated pre- } \\
\text { 2018): }\end{array}$ & $\begin{array}{l}\text { Year } \\
\text { Collected: }\end{array}$ & $\begin{array}{l}\text { Palmer } \\
\text { Drought } \\
\text { Index: }\end{array}$ & $\begin{array}{l}\text { Estimated } \\
\text { Elevation } \\
\text { (in } \mathrm{ft} \text { ): }\end{array}$ & $\begin{array}{l}\text { Average } \\
\text { Microbial } \\
\text { Richness: }\end{array}$ & $\begin{array}{l}\text { Total } \\
\text { Snails: }\end{array}$ \\
\hline $\begin{array}{l}\text { Mountain } \\
\text { Research } \\
\text { Station (MRS) }\end{array}$ & $\begin{array}{l}40.0314420 \\
-105.5394289\end{array}$ & 2018 & $\begin{array}{l}\text { Severe } \\
\text { Drought }\end{array}$ & 9675 & 483.36 & 15 \\
\hline Vail & $\begin{array}{l}\text { 39.6449151, } \\
-106.3146577\end{array}$ & 2018 & $\begin{array}{l}\text { Extreme } \\
\text { Drought }\end{array}$ & 8353 & 492.84 & 34 \\
\hline Frisco & $\begin{array}{l}39.5752723 \\
-106.1181343\end{array}$ & 2018 & $\begin{array}{l}\text { Extreme } \\
\text { Drought }\end{array}$ & 9298 & 425.36 & 8 \\
\hline $\begin{array}{l}\text { Steamboat } \\
\text { Springs } \\
(2004)\end{array}$ & $\begin{array}{l}\text { 40.4817935, } \\
-106.8245969\end{array}$ & 2004 & $\begin{array}{l}\text { Severe } \\
\text { Drought }\end{array}$ & 6505 & 315.91 & 13 \\
\hline Glen Eden & $\begin{array}{l}40.748779 \\
-106.843133\end{array}$ & 2004 & $\begin{array}{l}\text { Severe } \\
\text { Drought }\end{array}$ & 7513 & 288.29 & 7 \\
\hline Silverthorne & $\begin{array}{l}39.8356 \\
-106.31891\end{array}$ & 2003 & Mid-Range & 8586 & 493.33 & 3 \\
\hline Pagoda & $\begin{array}{l}\text { 40.321214, } \\
-107.344139\end{array}$ & 2000 & $\begin{array}{l}\text { Severe } \\
\text { Drought }\end{array}$ & 7008 & 122.50 & 2 \\
\hline Redstone & $\begin{array}{l}39.199847 \\
-107.232233\end{array}$ & 1982 & Very Moist & 7159 & 379.89 & 9 \\
\hline Telluride & $\begin{array}{l}37.934889, \\
-107.799037\end{array}$ & 1982 & Very Moist & 8809 & 459.25 & 4 \\
\hline $\begin{array}{l}\text { Steamboat } \\
\text { Springs } \\
(1980)\end{array}$ & $\begin{array}{l}40.472242 \\
-106.873814\end{array}$ & 1980 & Mid-Range & 8192 & 231.50 & 2 \\
\hline Rifle & $\begin{array}{l}39.727398 \\
-107.68823\end{array}$ & 1974 & $\begin{array}{l}\text { Moderate } \\
\text { Drought }\end{array}$ & 9583 & 592.50 & 4 \\
\hline $\begin{array}{l}\text { Glenwood } \\
\text { Springs }\end{array}$ & $\begin{array}{l}39.577612 \\
-107.369814\end{array}$ & 1974 & $\begin{array}{l}\text { Moderate } \\
\text { Drought }\end{array}$ & 6141 & 496.18 & 11 \\
\hline Boulder & $\begin{array}{l}39.929963 \\
-105.293328\end{array}$ & 1920 & $\begin{array}{l}\text { Moderately } \\
\text { Moist }\end{array}$ & 6237 & 245.33 & 3 \\
\hline
\end{tabular}


In the summer months of 2018 (between July and September) when terrestrial snails of the Rockies are most active, we collected fresh, living samples of Oreohelix strigosa from three locations within the Colorado Front Range: (1) the University of Colorado Mountain Research Station in Ward, Colorado (MRS), (2) the 10-mile trail in Frisco, Colorado and (3) the Gore Valley trail in Vail, Colorado. These freshly collected samples from 2018 ( $N=57$ ) included 15 from the MRS, 8 from Frisco, and 34 from Vail (Table 1). We used a qualitative collection method to collect specimens for this study, in accordance with Chalifour \& Li, 2021 [20] and Coppolino 2010 [23]. All collections were taken with the appropriate permitting for invertebrates. See Figure 2 for a complete map of collection localities and corresponding years.

Live snails were first drowned in distilled water and preserved in $95 \%$ ethanol for 24 hours, then transferred to and kept in $80 \%$ ethanol for permanent preservation as they were extracted, in accordance with CUMNH policies. This methodology has worked well on $O$. strigosa to increase plasticity in the tissue and ease of removal of the whole body from the shell for precise gut dissections. This is also consistent with the methodology used by some previous researchers who have deposited $O$. strigosa specimens in to the CUMNH collections.

Table 2. Breakdown of short-term treatment sample sizes within 2018 snails.

\begin{tabular}{|llllll|}
\hline Location: & Fresh: & 1 Month: & 6 Month: & 9 Month: & Starved: \\
\hline Mountain Research Station & 2 & 3 & 4 & 5 & 1 \\
\hline Vail & 6 & 6 & 9 & 10 & 3 \\
\hline Frisco & 2 & 1 & 1 & 2 & 2 \\
& & & & & \\
\hline Total: & 11 & 10 & 14 & 17 & 6 \\
\hline
\end{tabular}

Samples classified as "fresh" underwent no preservation and were extracted immediately after sacrifice. Periodically at ethanol preservation lengths of one, six, and nine months, we extracted gut tissue from a subset of each locality population. This resulted in 11 fresh samples, 10 one-month preserved samples, 14 six-month preserved samples, and 17 nine-month preserved samples (Table 2). Additionally, snails from each 2018 locality (a total of six samples) were kept under starvation conditions directly after collection with a natural photoperiod for approximately one week. They were given no food supplement of any kind. These starved snails were then sacrificed in the same manner as the other live snails.

\section{Microbial DNA Extraction}

All dissections in this study were performed aseptically, using sterile instruments. The soft body of the snail was removed by using fine forceps to gently pull the entire body out by the foot. In some cases, particularly of the older specimens, the soft body tissue had become too tough and could not be pulled out by the foot, so the apex of the shells was carefully broken and removed to expose the soft body, and 
then, the whole soft body was removed from the shells using forceps. The digestive tract was carefully isolated from the body, and a portion of gut was collected (Fig. 1C). We then re-preserved all shell and body parts individually in $80 \%$ ethanol after the necessary tissue was removed and re-catalogued these in the CUMNH collection accordingly.

We extracted genomic DNA from the snail gut tissue using the E.Z.N.A. Mollusk DNA Extraction kit according to the manufacturer's instructions (Omega BioTek, Norcross, GA, USA), as shown by our preliminary trials to be the optimal kit for microbial extraction of mollusk tissue by measures of DNA concentration. The V4 hypervariable region of the $16 \mathrm{~S}$ rRNA gene was amplified by PCR with the 515F/806R primer pair modified to include Illumina adapters and appropriate error-correcting barcodes, as comparable with other microbiome studies [24]. PCR amplification protocol was taken from the Earth Microbiome Project protocol for 515F/806R [24]. Library preparation and sequencing was facilitated by the Center for Microbial Exploration at the University of Colorado Boulder. 150 bp single indexed pairedend reads were generated on an Illumina Miseq platform PE300 (Illumina Corporation, San Diego, CA, USA) using a 2-by-150-bp paired end chemistry with the MiSeq V2 300-cycle kit (Illumina, San Diego, CA, USA). Samples were sequenced on one Illumina MiSeq run at the University of Colorado Next-Generation Sequencing Facility through BioFrontiers (Boulder, CO, USA).

Data were processed using the USEARCH10 pipeline [25] and the same processing as used in Chalifour \& Li 2021 [20]. Reads were merged with a minimum overlap of 16 bp (usearch8 -fastq_mergepairs). Trimmed reads were quality filtered with a max error rate of 1.0 (usearch10 -fastq_filter; $96.2 \%$ passed). Unique sequences were identified using usearch10 -fastx_uniques which clustered as 99\% 16S rRNA gene operational taxonomic units (OTUs) with usearch10 -cluster_otus uniques.fa. OTUs were classified taxonomically using the GreenGenes 13_8 database [26]. We removed OTUs that were classified as mitochondria or chloroplasts, and we did not use any samples that yielded fewer than 2,000 reads per sample. Six of the 112 snail gut samples (four 2018 samples, two 2004 samples) failed to meet this threshold for sequencing depth and were excluded from downstream analyses. Available data may be found at $[27,28]$.

\section{Statistical Analyses}

Data analysis was completed with R statistical software [29]. In sum, we examined community composition differences between our major treatment groups (i.e. explanatory variables), including the year collected, short-term preservation treatments, and location; as well as how several other ecological factors impact the community compositions. Once we determined how different the community compositions were, we identified which specific microbial taxa were driving the differences, using indicator species analyses. We then examined how microbial richness was affected by several explanatory variables, including year, short-term preservation treatment, and location. We also investigated species evenness and Shannon index as factors of microbial richness.

To evaluate and visualize the taxonomic makeup of our treatment groups, we ran Kruskal-Wallis tests comparing relative abundances of bacterial families across both years collected and short-term 
preservation treatments using the "taxa_summary_by_sample_type" function in \{MCToolsR\} and visualized the taxonomic compositions with the "plot_taxa_bars" function in \{MCToolsR\} [30].

Snail gut microbial compositional differences were assessed using a non-metric multidimensional scaling analysis (NMDS) based on year preserved, the short-term treatments of 2018 samples, and location. We used a Bray-Curtis distance matrix with a multiple regression analysis of all snails' gut microbiomes using microbial community diversity as the dependent variable for each explanatory variable (year, short-term preservation treatment, location). The NMDS allows us to visualize differences between gut community compositions based on groupings but does not give an indication of significant differences. Therefore, we subsequently used a permutation analysis of variance (PERMANOVA), a nonparametric test similar to an analysis of variance (ANOVA), to test for significant differences in microbial compositions among different treatment groups ("adonis2" function in \{vegan\} package) [31].

As part of the PERMANOVAs, we also explored how environmental factors that may impact gastropod physiology affect the gut microbiome compositions. The environmental factors included Palmer drought index (a measure of dryness based on precipitation and temperature) and elevation. We used the historic Palmer drought indices maps from the National Oceanic and Atmospheric Administration (NOAA) [32] to find the Palmer drought index for the month and year of the geographic region where each sample was collected. We used The National Map from the United States Geological Survey (USGS) [33] to estimate elevation for the location collected based on estimated latitude and longitude.

As geographic distances can be a major factor in shaping the microbial compositions of other animal microbiomes, we also ran a Mantel test to examine if there was any correlation between snail population geographic distance and microbial community similarity. The test was run in the \{vegan\} and \{geosphere\} $\mathrm{R}$ packages and tested for correlation between a geographic distance matrix of the Haversine distances of our estimated latitudes and longitudes and bacterial species abundance Bray-Curtis dissimilarity matrix [31, 34]. We used the \{ggmap\} R package [35] to plot collection points in Figure 2.

After determining where microbial compositions across these treatment groups significantly differed, we identified which specific bacterial species were making up the core microbiome across all samples and driving differences between groups. We used the "return_top_taxa" function of the $\{$ MCToolsR $\}$ package to initially discern which taxa were most prevalent across all snail gut samples, and give insight into the core microbiome [30]. To determine which bacterial species were associated with our different treatment groups, we used a similarity percentage (SIMPER) test using the "simper" function of the \{vegan\} package [36]. This test performs pairwise comparisons of treatment groups and finds the contribution of each bacterial species to the average between-group Bray-Curtis dissimilarity. We followed the SIMPER with a multilevel analysis of pattern (multipatt) using the "multpatt" function of the \{indicspecies\} package [37]. The mulitpatt shows bacterial species that are significantly associated to treatment groups, or treatment group combinations.

We tested not only how microbiome composition changed across these explanatory variables, but also how microbial richness changed. We used linear models to determine if year and the short-term treatment 
groups were significant predictors of microbial richness. As the response variable of microbial richness was non-normally distributed, and the relationship between microbial richness and collection year was non-linear, we used a negative binomial generalized linear model (see equations below) to test both the long-term preservation data (Equation 1) and the 2018 short-term preservation data (Equation 2). As there were no samples from every Palmer Drought Index taken at every time point, we could not determine how OTU richness was expected to change for a given index across the 98-year time range, so we used a separate negative binomial generalized linear model to test the indices from the long-term data (Equation 3). Negative binomial generalized linear models using the R package \{MASS\} were used to model microbial richness as a function of Palmer drought index, and of the fixed covariates: year, location, and elevation, and short-term preservation treatment and location. We also tested log-transformed and Poisson distribution generalized linear models along with the negative binomial generalized linear model against a null model to determine which model had the best fit. A Poisson distribution did not describe this data well because although microbial richness was still discrete, count data, the data was overdispersed as the variance greatly exceeded the mean. The AIC (Akaike information criterion) score of the negative binomial model was lowest, and therefore was the test chosen. Relevant predictors and their corresponding coefficient values and $p$-values are reported in Tables 3 and 4 .

\section{[Equation 1]}

Long-term Richness $=a+\beta_{1}($ Year Collected $)+\beta_{2}($ Location $)+\beta_{3}($ Elevation $)+$ error

\section{[Equation 2\}}

Short-term Richness $=a+\beta_{1}($ Short Term Treatment $)+\beta_{2}($ Location $)+$ error

\section{[Equation 3]}

Richness $=a+\beta_{1}($ Palmer Drought Index $)+$ error

Along with microbial richness, we also looked at evenness and Shannon index. We used Kruskal-Wallis tests to examine how evenness and Shannon indices changed based on year and short-term preservation treatment.

\section{Results}

Taxonomic Composition of Gut Bacterial Community

The microbiome composition of Oreohelix strigosa historical museum samples proved to be highly diverse. In total, there were 4,788,732 reads across all non-filtered out samples. The average number of reads per snail gut was $45,176.72$. The identified OTUs belonged to 37 unique phyla, 233 families, and 446 genera. 
Three OTUs were found in $100 \%$ of gut samples, and accounted for 1,534,035 reads, or roughly $32 \%$ of all reads. These were OTU_10 and OTU_9 (both members of bacterial family Enterobacteriaceae), and OTU_17 (Sphingomonas sp.). These three core taxa were also found in the top taxa across all samples. In $90 \%$ of gut samples, 22 OTUs accounted for 2,629,397 (55\%) reads. In $80 \%$ of gut samples, 39 OTUs accounted for 2,843,219 (59\%) reads. Other top taxa included Sphingobacterium faecium, Serratia sp., Lactococcus sp., Rahnella aquatilis, Spirosoma sp., Yersinia sp., Enterococcus sp., Spingomonas sp., and other Enterobacteriaceae members,

In total, historic samples and 2018 samples shared 3,603 OTUs. There were 1,852 OTUs unique to historic samples, and 1,356 OTUs unique to 2018 samples. These common OTUs largely came from phylum Proteobacteria, which accounted for roughly $41 \%$ of OTUs, followed by Bacteroidetes (16\%) and Actinobacteria (14\%). At the family level, the family with the most OTUs that was identifiable was Chinophagaceae, followed by Sphingomonadaceae and Chthoniobacteraceae.

At the family level, most collection years did not show significant differences of bacterial relative abundances (Fig. 3A). Kruskal-Wallis tests showed Enterobacteriaceae abundance was much lower in 1980 samples than other years at 0.3\%, and much higher in 2000 samples at $82 \%$. Samples from 2004 showed significantly higher abundances of Streptococcaeceae and Sphingobacteriaceae.

Short-term treatments also showed significant differences between relative abundances of bacterial families (Fig. 3B). Snails from the fresh treatment had significantly lower levels of Enterobacteriaceae, and higher levels of Chitinophagaeceae than the other treatments. Starved snails had significantly lower levels of Sphingobacteriaceae than the other treatments.

\section{Microbial Composition}

Across the entire dataset, location explained more variation in microbial communities than year. When controlling for location, the year 0 . strigosa samples were collected was a significant predictor of microbiome composition, but only explained $2.25 \%$ of variability between samples (Fig. 4A). In contrast, when controlling for year, location was again a significant predictor and explained $12.38 \%$ of variability between samples (Fig. 4B).

Within the 2018 dataset, short-term preservation treatment (including fresh, starved, 1 month, 6 month, and 9 month treatments separately) controlling for location was a significant predictor and explained $19.56 \%$ of variation (Fig. 4C). Location was also a significant predictor of microbiome composition and explained $16.86 \%$ of the variation between microbial community compositions when controlling for shortterm preservation treatment (Fig. 4D). More specifically, when categorizing short preservation treatments binarily as either preserved or not preserved, the presence of preservation explained much less (9.59\%) of the variation as location (17.15\%).

We also investigated other parameters that might explain some of the variation across gut community compositions. When controlling for year, the Palmer drought index was a significant predictor of

Page 10/29 
microbiome composition, but only explained $4.60 \%$ of the variation between sample compositions. When controlling for location, the Palmer drought index was a significant predictor of microbiome composition, but only explained $2.23 \%$ of the variation between sample compositions. Elevation of the collection site similarly was a significant predictor that explained very little of the variation across communities, only explaining $2.29 \%$ when controlling for year, and $2.64 \%$ when controlling for location.

A Mantel test comparing geographic distance and species abundance dissimilarity showed that the physical distances between all samples was significantly correlated with Bray-Curtis dissimilarity (Mantel statistic $\mathrm{R}=0.24, p$-value $<0.001)$. As samples became more dissimilar in geographic location, they also became more dissimilar in microbial community composition. This result holds true when analyzing microbial community composition from only 2018 samples, again, the geographic distance matrix has a moderate, but highly significant relationship with the species Bray-Curtis dissimilarity matrix (Mantel statistic $\mathrm{R}=0.27, p$-value $<0.001$ ).

\section{Indicator Species Analysis}

The top five taxa that contributed to differences between snails collected in 1920 and snails collected in 2018 were OTU_9 and OTU_10 (both members of family Enterobacteriaceae), OTU_1 (Sphingobacterium faecium), 0TU_27 (Pseudomonas sp.), and OTU_64 (Delftia sp.). The Enterobacteriaceae members and S. faecium dominated the 2018 samples over the 1920 samples, while the opposite was true for the Pseudomonas sp. and Delftia sp.

The top five taxa that contributed to differences between snails that underwent 9 months of preservation and fresh snails were OTU_9 and OTU_10 (both members of family Enterobacteriaceae), OTU_13 (a member of family Sphingobacteriaceae), OTU_1 (Sphingobacterium faecium), and OTU_18 (Spirosoma sp.). The Enterobacteriaceae members and $S$. faecium were more common in the 9 month samples than the fresh samples, while the opposite was true for the Spingobacteriaceae and Spirosoma sp. When simply looking at preserved versus unpreserved specimens from 2018 , four out of five indicator species remained the same (OTU_9, OTU_10, OTU_1, and OTU_13), with the fifth being OTU_4 (a member of Verrucomicrobiaceae). Again, the Enterobacteriaceae members and $S$. faecium were more common across the preservation treatments ( 1 month, 6 month, and 9 month), and the Spingobacteriaceae and Verrucomicrobiaceae were more common in the unpreserved treatments (starved and fresh).

We also ran a multilevel pattern analysis (multipatt) to determine which bacterial species can be used as indicators of certain treatment groups. When looking at year, there were 26 taxa associated to the year 1920,6 taxa to 1974,127 taxa to 1980,4 taxa to 1982,16 taxa to 2000,85 taxa to 2003,5 taxa to 2004 , and 12 taxa to 2018 .

Members of Cerasicoccaceae, Chitinophagaceae, Flavobacterium succinicans, and another Flavobacterium sp. were highly significantly ( $p$-value< 0.01) associated with being from 2018 . Bradyrhizobium sp., Alicycliphilus sp., and Cloacibacterium sp. were also significant, most strongly 
associated with coming from 1920 samples. There were five species found exclusively in 1980 samples. No other year had taxa exclusively found in its group samples.

The multilevel pattern analysis for short term treatment groups showed that no species were specifically associated with snails kept in preservation for 9 months. Starved snails had the highest number of associated taxa at 302, followed by fresh snails at 203, 6 month preservation has 9 associated taxa and 1 month preservation had 6 associated taxa.

\section{Microbial Richness}

The average richness per gut sample was 434.55 bacterial species, with a maximum of 1,328 species and a minimum of 35 species.

A negative binomial generalized linear model was used on all museum and field samples from 19202018. Collection year was not a significant predictor of microbial richness across all samples ( $p$-value $=$ 0.35 , Table 3). Location was also not significant predictor when controlling for year (Table 3). Palmer drought index was not a significant predictor of microbial richness, apart from snails deriving from "moderately moist" seasons, which had significantly lowered richness $(\exp \beta=-0.64 ; p$-value $=0.05)$. Elevation was also not a significant predictor of microbial richness ( $\exp \beta<0.001 ; p$-value $=0.23)$.

Table 3. Negative binomial generalized linear model predictors, coefficients, and $p$-values for models containing samples across all years. Boulder was used as the model intercept location.

\begin{tabular}{|lll|}
\hline Predictor & Coefficient & P-value \\
\hline Year & 0.015 & 0.353 \\
\hline Location - Frisco & -0.967 & 0.559 \\
\hline Location - Glen Eden & -1.125 & 0.432 \\
\hline Location - Glenwood Springs & -0.124 & 0.896 \\
\hline Location - Mountain Research Station (MRS) & -0.822 & 0.619 \\
\hline Location - Pagoda & -1.888 & 0.178 \\
\hline Location - Redstone & -0.501 & 0.642 \\
\hline Location - Rifle & 0.043 & 0.965 \\
\hline Location - Silverthorne & -0.577 & 0.687 \\
\hline Location - Steamboat Springs & -0.981 & 0.470 \\
\hline Location - Telluride & -0.331 & 0.762 \\
\hline Location - Vail & -0.803 & 0.626 \\
\hline Elevation & $<0.001$ & 0.229 \\
\hline
\end{tabular}


A negative binomial generalized linear model was used on all field samples collected during the summer of 2018. For a fixed location, undergoing no preservation (as part of the fresh and starved treatments) was a significant factor in gut microbial richness (Fig. 5).

Undergoing no preservation prior to gut extraction as part of the fresh treatment increased microbial richness by 0.52 ( $p$-value $=0.03$, Table 4 ) for a given location, while undergoing the starvation treatment and no preservation increased richness by 0.64 ( $p$-value $=0.01$, Fig. 5 , Table 4$)$. There were non-significant effects of the other short-term preservation treatments, and there was no significant effect of location (Table 4).

Table 4

Negative binomial generalized linear model predictors, coefficients, and $p$-values for model containing only 2018 samples. * indicates significant values. Frisco, 1-month preservation was the model intercept.

\begin{tabular}{|lll|}
\hline Predictor & Coefficient & P-value \\
\hline Short-term Treatment - 6 Month & 0.328 & 0.109 \\
\hline Short-term Treatment - 9 Month & -0.113 & 0.573 \\
\hline Short-term Treatment - Fresh & 0.5152 & 0.030 * \\
\hline Short-term Treatment - Starved & 0.638 & 0.014 * \\
\hline Location - Mountain Research Station (MRS) & 0.127 & 0.577 \\
\hline Location - Vail & 0.205 & 0.307 \\
\hline
\end{tabular}

Kruskal-Wallis tests showed that evenness changed non-significantly across most years, except for pairwise differences between 2004 and 1920 ( $p$-value < 0.05), 2004 and 1974 ( $p$-value < 0.05), and 2018 and 1974 ( $p$-value $<0.05)$. Kruskal-Wallis tests showed that Shannon Indices changed non-significantly across most years, except for Shannon Indices in 2004 and 1974 which showed a significant pairwise difference $(p$-value $<0.001)$.

\section{Discussion}

Recent research has revealed a unique, stable microbiome in Oreohelix strigosa [20]. However, the effect of ethanol preservation on microbial composition within museum specimens over long periods of time has yet to be elucidated. In this study, we targeted the gut microbiome of $O$. strigosa that were historically collected (between 1920 and 2014), preserved in ethanol and stored in museum collections along with newly (2018) field collected $O$. strigosa used for short-term preservation treatments. Our results highlighted that long-term preservation does not significantly impact the makeup of the microbiome, in terms of alpha or beta diversity. Most variation occurs within the initial time frame of preservation, and then microbiome structure appears stable throughout time. Significant variation in community composition is attributed to factors other than preservation period, including geographic location. Other 
factors not addressed in this study, such as whether the samples were first fixed in formalin and then moved into ethanol preservation, percentage of ethanol used (e.g., $70 \%$ versus $95 \%$ ethanol), and temperature-controlled storage, as well as fluctuations like freeze-thaw cycles, may have a greater impact on microbiome makeup than storage time $[6,21,38]$.

\section{The Core Microbiome}

The core microbiome are bacteria that are always associated with a particular host species, regardless of diet, location, or other environmental variables. The core microbiome can provide insight into which microbes facilitate the host's ability to consume certain food items, or other life history processes. The core microbiome of $O$. strigosa found in this study is consistent with that found in Chalifour \& Li, 2021 [20]. They consisted of two members of family Enterobacteriaceae, and one unidentified Spingomonas species. These three OTUs made up nearly a third of all sequenced reads. Despite being collected across a broad temporal and geographic gradient within Colorado, our results suggest that there is a shared core microbiome across all these $O$. strigosa specimens. In addition, both historic and 2018 samples shared 3,603 OTUs, or around half of all sequenced OTUs, showing that there is stability across the 98-year period of gut microbiome residents.

Members of Enterobacteriaceae, Sphingobacteriaceae, and Sphingomonadaceae have all been associated with other snail species' gut microbiomes [17, 39, 40]. While associations between Oreohelix and their gut bacteria have barely been studied, their diet and habitats suggest that the microbiome may have important functional roles in cellulose breakdown. Among some of the representative 0 . strigosa microbiome species, Flacobacterium succinicans has been found in Daphnia guts [41], as well as soil and fresh water. Members of Roseomonas have been found in the gill and gastrointestinal microbiomes of Tilapia [42]. Stenotrophomonas maltophilia and other Stenotrophomonas species have been identified in bark beetle guts, and $S$. maltophilia in particular showed cellulolytic activity [43].

\section{Microbial Composition}

\section{Preservation and Gut Microbiome Community Composition}

Our results show that environmental factors are more likely to affect the microbiome composition than preservation factors. Long-term preservation seems to impact the makeup of the microbiome only marginally (Fig. 4A) compared with location which explained much more of the microbial composition variability (Fig. 4B) and does not significantly impact the richness of the microbiome (Fig. 5). Only 2.25\% of variation in gut bacterial diversity was explained by the year the snail samples were collected (Fig. 4A, $\mathrm{p}$-value $<0.001)$. In contrast, $12.38 \%$ of variation in microbiome composition was explained by the location the snail sample was collected (Fig. 4B, p-value < 0.001 ). So, samples from 50 years ago have just as much potential to answer biological questions as those from 10 years ago or less. Importantly, most samples, regardless of being historic or current, passed all quality control steps within the USEARCH pipeline [25], along with rarefication. This preliminary, but vital step, shows that next-generation sequencing technology can characterize bacterial communities of historic specimens as well as 
contemporary specimens. In other studies, sequencing platform variability has a negligible effect of microbiome makeup differences across samples, rather, storage conditions are far more important in driving differences [44].

Similar studies of museum specimens over shorter periods of time have been conducted with varying results. The longest study known to us is one by Neu et al., 2021, which investigated microbiome composition and richness of the ethanol-preserved marine bivalve, Donax gouldii, over the span of one decade [2]. As with our study, Neu et al. show that the preserved, historic tissue provided high quality information about temporal trends across the 11-year period. Importantly, these samples were preserved in $95 \%$ ethanol at 4 degrees Celsius, rather than in $80 \%$ ethanol at room temperature. Nonetheless, their result is consistent with our findings that historic samples can be effectively used over longer time scales to study animal microbiomes [2]. In agreement with our study, Neu et al. 2021 found microbiome composition and richness across the temporal range were stable, and there was a core microbiome found across all time points [2]. Another study of a smaller scale by Heindler et al. (2018) showed that preserved fish specimens had a very clear change in microbiome composition over time, but it is due to true biological shifts and not preservative biases [6].

Short-term preservation accounts for more of the variation between 2018 samples than location (Fig. 4C, D), but most changes happen between unpreserved and preserved treatments, rather than across each preservation time point. Location explained more of the variance when compared to the binary explanatory variable of the presence of preservation (whether a sample was preserved at all [1 month, 6 month, 9 month treatments], or never preserved [fresh and starved treatments]). In another short-term study only preserving specimens up to 60 days, the effect of preservation was also detected in their PERMANOVAs in explaining microbiome compositions [15]. However, this short-term study also tested different storage mediums (e.g. ethanol, freezing, RNA Later, etc.) and found that ethanol preservation was the most consistent in working well over time to preserve the microbiome and there was no difference between fresh and preserved insect samples; the effect of preservation was more prevalent in the other methodologies like RNALater. As ethanol was the most stable of the methods tested, this could explain why samples preserved long-term in ethanol solution showed stability in their composition, and samples preserved for the short-term monthly periods also remained more similar to each other than those not preserved at all.

As shown by short-term preservation studies $[13,14]$, initial method of fixation or preservation of the host specimen is impactful to the microbiome preservation. Microbial heterogeneity in the microbiome is the main factor that responds differently to varying types of preservation methods [45]. As microbiome technology has rapidly developed and become more advanced in recent years, sample preservation practices for this purpose were not considered for many older museum specimens, and as such, were based on tradition rather than experimentation to find the best practice to preserve microbial associations [46]. Therefore, the initial method of preservation may not be consistent over time and may be adding to the variability between individual samples or collected lots. 
Variation due to anesthetization method is likely contributing to the initial shifts of gut microbiome diversity immediately after preservation in this study. We drowned snails to anesthetize them before fixing them in ethanol, as it is the traditional procedure for snail sacrifice. This method may have caused snails to expel some amount of microbial species into the water that is then lost in every treatment downstream of this step, thus explaining why non-preserved treatments were more similar to each other and preserved treatments of any age were also more similar. One study postulates that drowning actually degrades DNA [47], however, there is considerable benefit in obtaining well-relaxed soft bodies. In our experience, well-relaxed bodies make removal from the shell less destructive and allow the body to come out in one piece, which is important for preserving the morphology of the snail and performing accurate dissections [48]. Additionally, a later study by Kruckenhauser et al. showed that drowning did not affect the DNA quality needed for successful PCR amplification and other DNA-based methods [48]. Therefore, if drowning or other relaxation methods (e.g., magnesium chloride) are necessary for preserving certain taxa, it might be valuable to sequences a few freshly collected individuals as a microbiome reference.

Although we believe ethanol was the only preservative used in our samples, in some older museum specimens, formalin was initially used to preserve specimens, starting in 1891 and gaining popularity into the first quarter of the twentieth century [49]. Formalin can cause crosslinking among DNA molecules, even resulting in depolymerization of the DNA $[6,50,51]$. Unfortunately, the initial preservative is not always documented in museum records. To allow future researchers to use museum samples for microbiome study, detailed descriptions of sampling and preservation procedures should be employed to reduce variability and error from preservation practices in samples.

\section{Location and Gut Microbiome Community Composition}

A Mantel test showed that geographic distances and Bray-Curtis dissimilarities were significantly correlated with one another ( $p$-value $<0.001)$. Though the $\mathrm{R}$ value is moderate $(0.24)$, this test does substantiate the idea that snail gut microbiome changes are partially driven by geographic distances. When controlling for year, location was a significant predictor of gut microbiome composition and explained $12.38 \%$ of variability between all samples, and $16.91 \%$ of variability in just 2018 samples.

In other systems, location also appears to account for significant differences in microbiome variation. Griffiths et al. [52] found significant results of a Mantel test between geographic distance matrices and microbiome distance matrices in the sponge Ircinia campana, and generally, geographic location has been shown in many sponges to drive intraspecific microbiome variation [52,53,54,55]. In another invertebrate, corals, both the microbiome and the pathobiome (pathogenic microbial assemblage) are dependent on geographic location [56]. Both honeybees and oysters show gut communities that differ based on sites, and in bees, among colonies within sites [57, 58].

Although geographic location appears to be a significant driver of a large proportion of the variation across samples, it is possible that this pattern is driven by other environmental factors that co-vary with location, such as habitat, temperature, drought level, or diet. For example, animal diets have been shown to be strongly correlated with the bacterial compositions present in their gut microbiomes [42]. Members

Page 16/29 
of the same host species originating from different habitats become exposed to distinct microbial communities and are then colonized by distinct gut microbial communities [19]. Shifts in diet happen regularly for wild animals, based on food availability, season, etc., and are strongly correlated with habitat and location [19]. For example, Black howler monkeys show distinct differences in gut community composition based on four habitats, each unique in what diets it enables [19]. Kohl et al. [59] found that diet had a significant effect on gut microbial community structure in wild lizards. Sea urchins also show a diet-specific bacterial community when larvae are cultured in specific locations [60]. This may also be common in snails, as in various other invertebrate host/bacterial symbiont systems, microbes are transmitted horizontally [61]. Many land snails use a generalist feeding strategy, and thus have evolved unique gut microbiomes to efficiently breakdown and use a variety of tough, cellulolytic, vegetative materials for their own nutrition and growth $[17,62]$. Some terrestrial snails are known to augment their gut microbiome through horizontal transmission, or the collection of environmental bacteria from their surrounding habitat through eating [17]. Thus, it is likely that each 0 . strigosa snail population harbors a gut microbiome that is unique to their specific habitat, and thus a geographically specific diet, regardless of how long it has been in preservation for.

Palmer drought index and elevation as explanatory variables did not significantly drive any changes in microbial composition. In other animals, drought can be a good indicator of resource abundance or restriction. In buffalo, shifts in gut microbiome composition and the enterotypes present are driven by resource restriction associated with drought conditions, also resulting in decreased number of microbial species present in the gut [63]. However, $O$. strigosa microbiomes do not show significant disturbances due to drought. This may be because these snails are more drought tolerant than other animal species; they regularly aestivate when conditions are too hot or dry, and so their microbiome may be more acclimated to shifts in habitat favorability [64]. In general, many snails are known to exist synergistically with drought, altering their feeding apparatus in unison with drought events [65] and have a high tolerance and survival rate to prolonged drying events [66].

\section{Indicator Species Analysis}

The indicator species present in varying treatment groups of $O$. strigosa are all common across other gastropod microbiomes. Members of Verrucomicrobia are found in planorbid snails, including Bulinus africanus and Helisoma duryi [67]. Members of Spirosoma have been characterized in the Hawaiian tree snail Achatinella mustelina [68]. Enterobacteriaceae are present in the land snails Achatina fulica, Cornu aspersum, Helix pomatia, and Helix aspersa and are hypothesized to take part in food fermentation in the gut $[17,38,69,70]$.

In general, the later years sampled (from 2000 through 2018) showed Palmer drought indexes of extreme or severe drought, while most moister years occurred in the 1980s and before. In these later years, the family Enterobacteriaceae are more prevalent than in earlier years (Fig. 3A). Members of Enterobacteriaceae have been found to have a significant seed germination-promoting effect and stimulate seedling growth in plant species inoculated with these strains under severe drought stress [71]. As Enterobacterstrains are drought tolerant and have a diverse growth range under stressful conditions, 
they may be more common in snail hosts that are also experiencing drought stress [72]. Snails may be feeding on plant matter that was assisted in growing by Enterobacteriaceae that are still present in the plant, thus being exogenously passed to the snail gut. Likewise, a member of family Cerasicoccaceae was associated to 2018 snails, which has also been associated with drastic environmental changes (such as changes in precipitation) in planktonic bacterial communities [38]. While there are shifts in indicator species for particular years, the overall microbial community compositions do not differ significantly among the years, explaining why drought index is not a significant driver. Similar results have been found in other systems. For example, thermally stressed barrel sponges show no significant community changes of the microbiome compared with non-stressed sponges [73]. There was however evidence of functional changes between these groups, with stressed sponges having lowered abundance of microbial photosynthetic proteins [73]. While the overall snail gut microbiome composition may stay similar throughout moist and drought years, there may be some key microbial players that do change in abundance in accordance with climate-induced shifts. Therefore, future studies should focus on understanding how ecological factors impact the abundance of different functional microbial groups, in addition to overall microbiome compositions.

\section{Microbial Richness}

Microbial richness shows a low amount of variation driven specifically by year. Consequently, community composition, rather than variation in richness, is responsible for the observed variation in the microbial communities across these snails. Elevation showed no significant effect on richness, and neither did Palmer drought index, apart from a significantly lowered richness in snails collected in moderately moist seasons. Overall, our findings support that long-term preservation allows for unbiased quantification of the microbial richness of the gut microbiome, as it remained stable over the 98 -year temporal range of this study.

We found that the initial transition from being non-preserved to preserved had the most impact on altering the richness of the microbiome. For the 2018 samples, significant differences seem to lie between the completely unpreserved treatments (fresh, starved) and the treatments with some level of preservation (1 month, 6 month, 9 month). For a fixed location, undergoing no preservation (as part of the fresh or starved treatments) was a significant factor in increasing gut microbial richness. This may be due to the method of fixation and preservation used, (e.g., drowning method, ethanol vs. formalin) as discussed in the microbial composition discussion above.

Nonsignificant differences between the richness of different communities could be due to host related factors. Varying diet composition, as discussed before, could explain the greater variation in richness seen when looking specifically at location. The stage of development of the host can also create variations in microbiome richness $[20,74]$. As all the snails used in this study were adults, we do not think development stage was a factor here, however, it should be considered in future studies of this nature. Host gut length has been suggested as a factor in shaping the microbiome and could also be considered in future studies [74]. Additionally, in other studies, host genotype can be extremely important in shaping 
the gut microbiome and should be considered when using museum specimens from multiple species [18, 75].

\section{Conclusions}

The use of advanced molecular technology offers a novel method to study microbial communities of ethanol-preserved museum specimens. In this study, we used next-gen high-throughput sequencing technique to investigate the bacterial diversity of museum specimens of the snail species $O$. strigosa across nearly a century of preservation age. We have demonstrated that specimens from all ages generated high quality sequencing data, and the snails' core microbiomes were stable across all museum specimens. Both long- and short-term preservation time explained little variation in the data, with other factors like location explaining more of the variation between samples. Further research is required to better characterize other potential driving factors of microbiome variability in $O$. strigosa, which include initial preservation and storage methodologies, especially for museum samples.

Microbiome preservation, or microbiome banking, is vital to conserving the unique amalgamation of microbes associated with a particular host. This knowledge can be useful downstream for determining how missing components of a native microbiome of a system can affect its immunity to disease, and survival potentials [45]. It is also useful when considering that many microbes can be specific to their host, so, when a host goes extinct, microbial diversity also shrinks [45]. The preservation of intact samples and collection of extracted DNA is important not only to study rare or extinct host specimens, but also because it is one way to study environmental and climatic variables on microbial communities in the past, or in the future. Additionally, microbiome studies of captive animals are often not representative of how the host and microbiome would interact in its natural habitat, so museum preserved wild animal specimens can provide a critical means of comparison [19]. There is no universal, single preservation method that works for all microbiome samples, therefore individual studies unique to systems are vital. Many natural history museums employ similar methods of ethanol preservation for their mollusk collections. By investigating the same species, preserved in the same method over time, we aimed to limit the preservative biases present to support that host preservation is intimately tied to microbiome preservation.

Our findings support the idea that researchers should not be deterred from using historic samples from museum collections. These results open the doors for using museum specimens to assess how microbiomes change over time and space in response to ecological variables. For example, in this study we were able to find the Palmer drought index for all samples and time periods. A comparative study of samples from extreme drought summers versus very moist summers could be done, along with any number of climatic variations using historic databases alongside historic specimens. This also enables worldwide microbiome sampling campaigns, without requiring new field work [76]. As most museum specimens are deposited whole, these snails were not previously dissected, which limited any access to the gut and further reduced variability across samples. 
In the future, similar studies need to be done on more diverse museum specimens and on various types of microbiomes (mucosal, fecal, whole body, etc.). Consistent methods of preservations are necessary to definitively reduce sources of variability across microbiome sample compositions. At least, consistent records of preservation techniques along with detailed locality notes are helpful for researchers conducting microbial ecology studies. It is similarly vital for ecologists to communicate closely with museum workers to create a coordinated approach for sample lending, destruction, and the return of usable DNA to the museum institution [77]. This study should encourage further use of museums as a resource for studying microbial ecology across time and space.

\section{Abbreviations}

AIC

Akaike information criterion

\section{ANOVA}

Analysis of variance

\section{CUMNH}

University of Colorado Museum of Natural History

\section{DNA}

Deoxyribonucleic acid

\section{MRS}

University of Colorado Mountain Research Station

\section{Multipatt}

Multilevel analysis of pattern

\section{NMDS}

Non-metric multi-dimensional scaling

\section{NOAA}

National Oceanic and Atmospheric Administration

\section{OTU}

Operational taxonomic unit

PCR

Polymerase chain reaction

\section{PERMANOVA}

Permutational analysis of variance

\section{rRNA}

Ribosomal ribonucleic acid

\section{SIMPER}

Similarity percentage

\section{USGS}

United States Geological Survey 


\section{Declarations}

\section{Ethics approval and consent to participate}

Not applicable.

\section{Consent for Publication}

Not applicable.

\section{Availability of Data and Materials:}

The data that support the findings of this study will be openly available in FigShare at http://doi.org/[doi] once the manuscript is accepted. Related metadata can be found at (FigShare Link 1) [27] and Supplementary Table 1 provides unique sample identifier tags that can be matched to both the deposited sequence data (FigShare Link 2) [28] and deposited metadata (FigShare Link 1) [27], along with all associated museum database information.

\section{Competing Interests:}

The authors declare that they have no competing interests.

\section{Funding:}

Funding for this project was provided by the Kurt Gerstle Fellowship from the Colorado Mountain Club Foundation; the Beverly Sears Graduate Student Grant from the University of Colorado Boulder; and the Museum Student Research Award from the University of Colorado Museum of Natural History.

\section{Authors' Information:}

Authors' Affiliations:

Department of Ecology and Evolutionary Biology, University of Colorado Boulder, 1900 Pleasant Street, 334 UCB, Boulder, CO, USA 80309

Bridget N. Chalifour \& Jingchun Li

Museum of Natural History, University of Colorado Boulder, 265 UCB, Boulder, CO, USA 80309

Leanne E. Elder \& Jingchun Li

Authors' Contributions:

B.N.C. and J.L. conceived the initial study. B.N.C. designed, implemented, and coordinated the field work portion of the study. B.N.C. and J.L. designed and implemented the laboratory portion of the study. B.N.C. developed computational pipelines and led data analysis and interpretation, with assistance from all 
authors. L.E.E. led all curatorial work associated with the project. B.N.C. drafted the manuscript, with all authors contributing to writing and revisions.

Corresponding Author:

Correspondence to Bridget Chalifour (bridget.chalifour@colorado.edu).

\section{Acknowledgments:}

We would like to thank the Center for Microbial Exploration at the University of Colorado Boulder, Dr. Noah Fierer for guidance with protocol design, and Matthew Gebert for assistance with sample preparation and processing. We thank the BioFrontiers Institute Next-Gen Sequencing Core Facility, which performed the Illumina sequencing and library construction. We would like to thank the Li lab group for providing comments on the drafts of this manuscript. We would also like to thank the University of Colorado Mountain Research Station, along with the University of Colorado Museum of Natural History for use of their historic specimens and databased records. This work was supported by the Kurt Gerstle Fellowship, the Colorado Mountain Club Foundation; the Beverly Sears Graduate Student Grant, University of Colorado Boulder; and the Museum Student Research Award, University of Colorado Museum of Natural History.

\section{References}

1. McFall-Ngai M, Hadfield MG, Bosch TC, Carey HV, Domazet-Lošo T, Douglas AE, et al. Animals in a bacterial world, a new imperative for the life sciences. PNAS. 2013;110(9):3229-3236.

2. Neu AT, Hughes IV, Allen EE, Roy K. Decade-scale stability and change in a marine bivalve microbiome. Molec Ecol. 2021;30(5):1237-1250.

3. West AG, Waite DW, Deines P, Bourne DG, Digby A, McKenzie VJ, et al. The microbiome in threatened species conservation. Biol Conserv. 2019;229:85-98.

4. Komatsu KJ, Avolio ML, Lemoine NP, Isbell F, Grman E, Houseman GR, et al. Global change effects on plant communities are magnified by time and the number of global change factors imposed. PNAS. 2019;116(36):17867-17873.

5. Yeates DK, Zwick A, Mikheyev AS. Museums are biobanks: unlocking the genetic potential of the three billion specimens in the world's biological collections. Curr Opin Insect Sci. 2016;18:83-88.

6. Heindler FM, Christiansen H, Frédérich B, Dettaï A, Lepoint G, Maes GE, et al. Historical DNA Metabarcoding of the Prey and Microbiome of Trematomid Fishes Using Museum Samples. Front Ecol Evol. 2018;6:e5537.

7. Bahrndorff S, Alemu T, Alemneh T, Lund Nielsen J. The microbiome of animals: implications for conservation biology. Int J Genomics. 2016.

8. Jaksch K, Eschner A, Rintelen TV, Haring E. DNA analysis of molluscs from a museum wet collection: a comparison of different extraction methods. BMC Res Notes. 2016;9(1):1-12. 
9. Gross BA. Evaluating the effects of three preservation methods on DNA quality and morphology of museum specimens of the American Alligator (Alligator mississippiensis) (Doctoral dissertation). 2017.

10. Huang W, Xie X, Liang X, Wang X, Chen X. Effects of different pretreatments of DNA extraction from dried specimens of ladybird beetles (Coleoptera: Coccinellidae). Insects. 2019;10(4):91.

11. Mandrioli M, Borsatti F, Mola L. Factors affecting DNA preservation from museum-collected lepidopteran specimens. Entomol Exp. 2006;120(3):239-244.

12. Sproul JS, Maddison DR. Sequencing historical specimens: successful preparation of small specimens with low amounts of degraded DNA. Molec Ecol Res. 2017;17(6):1183-1201.

13. Chakraborty A, Sakai M, Iwatsuki Y. Museum fish specimens and molecular taxonomy: a comparative study on DNA extraction protocols and preservation techniques. J Appl Ichthyol. 2006;22(2):160-166.

14. Hammer TJ, Dickerson JC, Fierer N. Evidence-based recommendations on storing and handling specimens for analyses of insect microbiota. PeerJ. 2015;3: e1190.

15. Yang ZW, Men Y, Zhang J, Liu ZH, Luo JY, Wang YH, et al. Evaluation of Sample Preservation Approaches for Better Insect Microbiome Research According to Next-Generation and ThirdGeneration Sequencing. Microb Ecol. 2021;82(4):971-980.

16. Sinclair-Winters CM. Upstream or downstream? Population structure of the land snail Ventridens ligera (Say, 1821) in the Potomac River drainage basin. J Molluscan Stud. 2014;80(3):280-285.

17. Dar MA, Pawar KD, Pandit RS. Gut Microbiome Analysis of Snails: A Biotechnological Approach. In: Ray S editor. Organismal and Molecular Malacology. Rijeka: InTech; 2017. p. 189-217.

18. Chalifour B, Li J. A Review of the Molluscan Microbiome: Ecology, Methodology and Future. Malacologia. 2021;63(2):285-304.

19. Amato KR. Co-evolution in context: the importance of studying gut microbiomes in wild animals. Microbiome Sci Med. 2013;1(1).

20. Chalifour B, Li J. Characterization of the gut microbiome in wild rocky mountainsnails (Oreohelix strigosa). Animal Microbiome. 2021;3(1):49.

21. Song SJ, Amir A, Metcalf JL, Amato KR, Xu ZZ, Humphrey G, Knight R. Preservation methods differ in fecal microbiome stability, affecting suitability for field studies. MSystems. 2016;1(3):e00021-16.

22. Rubin BE, Gibbons SM, Kennedy S, Hampton-Marcell J, Owens S, Gilbert JA. Investigating the impact of storage conditions on microbial community composition in soil samples. PloS One. 2013;8(7):e70460.

23. Coppolino ML. Strategies for collecting land snails and their impact on conservation planning. Am Malacol Bull. 2010;28(2):97-103.

24. Thompson LR, Sanders JG, McDonald D, Amir A, Ladau J, Locey KJ, et al. A communal catalogue reveals Earth's multiscale microbial diversity. Nature. 2017;551:457-463. 
25. Edgar RC. Search and clustering orders of magnitude faster than BLAST. Bioinformatics. 2010;26(19):2460-1.

26. McDonald D, Price MN, Goodrich J, Nawrocki EP, DeSantis TZ, Probst A, et al. An improved Greengenes taxonomy with explicit ranks for ecological and evolutionary analyses of bacteria and archaea. ISME J. 2012;6:610-8.

27. [dataset] Chalifour, B.N., Elder, L.E., Li, J. (2021) OTU Table O. strigosa Preservation; FigShare; DOI: To be added upon acceptance.

28. [dataset] Chalifour, B.N., Elder, L.E., Li, J. (2021) Mapping File O. strigosa Preservation; FigShare; DOI: To be added upon acceptance.

29. R Core Team. R: A language and environment for statistical computing. R Foundation for Statistical Computing, Vienna, Austria. 2019. https://www.R-project.org/.

30. Leff J. MCToolsR. 2014. https://github.com/leffj/mctoolsr/.

31. Oksanen JF, Blanchet G, Friendly M, Kindt R, Legendre P, McGlinn D, et al. vegan: Community Ecology Package. $\mathrm{R}$ package version 2.5-6. 2019. https://CRAN.R- project.org/package = vegan .

32. Vose RS, Applequist S, Squires M, Durre I, Menne MJ, Williams Jr CN, et al. Improved historical temperature and precipitation time series for US climate divisions. J Appl Meteorol Climatol. 2014;53(5):1232-1251.

33. U.S. Geological Survey. USGS TNM Topography 2019. https://apps.nationalmap.gov/viewer/

34. Hijmans RJ. geosphere: Spherical Trigonometry. 2019. https://CRAN.R- project.org/package = geosphere

35. Kahle D, Wickham H. ggmap: Spatial Visualization with ggplot2. R J. 2013;5(1):144-161.

36. Clarke KR. Non-parametric multivariate analyses of changes in community structure. Austral Ecol. 1993;18(1):117-143.

37. Cáceres MD, Legendre P. Associations between species and groups of sites: indices and statistical inference. Ecology, 2009;90(12):3566-3574.

38. Ma T, Jiang Y, Elbehery AH, Blank S, Kurmayer R, Deng L. Resilience of planktonic bacterial community structure in response to short-term weather deterioration during the growing season in an alpine lake. Hydrobiologia. 2020;847(2):535-548.

39. Koleva Z, Kizheva Y, Tishkov S, Dedov I, Kirova E, Stefanova P, et al. Dynamics of bacterial community in the gut of Cornu aspersum. J. BioSci. Biotechnol. 2015;4(3):263-269.

40. Portet A, Toulza E, Lokmer A, Huot C, Duval D, Galinier R, et al. Infection of the Biomphalaria glabrata vector snail by Schistosoma mansoni parasites drives snail microbiota dysbiosis. BioRxiv. 2019;386623.

41. Freese HM, Schink B. Composition and stability of the microbial community inside the digestive tract of the aquatic crustacean Daphnia magna. Microb Ecol. 2011;62(4):882.

42. Wu Z, Zhang Q, Lin Y, Hao J, Wang S, Zhang J, Li A. Taxonomic and Functional Characteristics of the Gill and Gastrointestinal Microbiota and Its Correlation with Intestinal Metabolites in NEW GIFT 
Strain of Farmed Adult Nile Tilapia (Oreochromis niloticus). Microorganisms. 2021;9(3):617.

43. Morales-Jiménez J, Zúñiga G, Ramírez-Saad HC, Hernández-Rodríguez C. Gut- associated bacteria throughout the life cycle of the bark beetle Dendroctonus rhizophagus Thomas and Bright (Curculionidae: Scolytinae) and their cellulolytic activities. Microb Ecol. 2012;64(1):268-278.

44. Ma J, Sheng L, Hong Y, Xi C, Gu Y, Zheng N, et al. Variations of gut microbiome profile under different storage conditions and preservation periods: a multi- dimensional evaluation. Front Microbiol. 2020;11:972.

45. Prakash O, Nimonkar Y, Desai D. A Recent Overview of Microbes and Microbiome Preservation. Indian J Microbiol. 2020;60(3): 297-309

46. Pierson TW, Kieran TJ, Clause AG, Castleberry NL. Preservation-induced morphological change in salamanders and failed DNA extraction from a decades-old museum specimen: implications for Plethodon ainsworthi. J Herpetol. 2020;54(2):137-143.

47. Schander C, Hagnell J. Death by drowning degrades DNA. J Moll Stud. 2003;69(4):387-388.

48. Kruckenhauser L, Harl J, Sattmann H. Optimized drowning procedures for pulmonate land snails allowing subsequent DNA analysis and anatomical dissections. Annalen des Naturhistorischen Museums in Wien. Serie B für Botanik und Zoologie, 2010; p: 173-175.

49. Herbin M. La conservation des collections en fluide. Approche historique et conservatoire. In CeROArt. Conservation, exposition, Restauration d'Objets d'Art (No. HS). Association CeROArt asbl. 2013.

50. Thomas Jr. CA, Doty P. The mild acidic degradation of desoxyribose nucleic acid. J Am Chem Soc. 1956;78(9):1854-1860.

51. Geiduschek EP. On the reversibility of the acid denaturation of sodium desoxyribose nucleate. J Polym Sci. 1958;31(122):67-75.

52. Griffiths SM, Antwis RE, Lenzi L, Lucaci A, Behringer DC, Butler IV, et al. Host genetics and geography influence microbiome composition in the sponge Ircinia campana. J Anim Ecol. 2019;88(11):16841695.

53. Fiore CL, Jarett JK, Lesser MP. Symbiotic prokaryotic communities from different populations of the giant barrel sponge, Xestospongia muta. Microbiologyopen. 2013;2(6):938-952.

54. Luter HM, Widder S, Botté ES, Wahab MA, Whalan S, Moitinho-Silva L, et al. Biogeographic variation in the microbiome of the ecologically important sponge, Carteriospongia foliascens. PeerJ. 2015;3:e1435.

55. Swierts T, Cleary DFR, de Voogd NJ. Prokaryotic communities of Indo-Pacific giant barrel sponges are more strongly influenced by geography than host phylogeny. FEMS Microbiol Ecol. 2018;94(12):194.

56. Rubio-Portillo E, Kersting DK, Linares C, Ramos-Esplá AA, Antón J. Biogeographic differences in the microbiome and pathobiome of the coral Cladocora caespitosa in the Western Mediterranean Sea. Frontiers in Microbiology, 2018;9:22. 
57. King GM, Judd C, Kuske CR, Smith C. Analysis of stomach and gut microbiomes of the eastern oyster (Crassostrea virginica) from coastal Louisiana, USA. PLoS one. 2012;7(12):e51475.

58. Moran NA, Hansen AK, Powell JE, Sabree ZL. Distinctive gut microbiota of honeybees assessed using deep sampling from individual worker bees. PloS one. 2012;7(4):e36393.

59. Kohl KD, Brun A, Magallanes M, Brinkerhoff J, Laspiur A, Acosta JC, et al. Gut microbial ecology of lizards: insights into diversity in the wild, effects of captivity, variation across gut regions and transmission. Molec Ecol. 2017;26(4):1175-1189.

60. Carrier TJ, Dupont S, Reitzel AM. Geographic location and food availability offer differing levels of influence on the bacterial communities associated with larval sea urchins. FEMS Microbiol Ecol. 2019;95(8):103.

61. Chrostek E, Pelz-Stelinski K, Hurst GD, Hughes GL. Horizontal transmission of intracellular insect symbionts via plants. Front Microbiol. 2017;8:2237.

62. Burch JB, Pearce TA. Terrestrial gastropoda. In: Soil biology guide. 1990. p. 201-309.

63. Couch CE, Stagaman K, Spaan RS, Combrink HJ, Sharpton TJ, Beechler BR, et al. Diet and gut microbiome enterotype are associated at the population level in African buffalo. Nat Commun. 2021;12(1):1-11.

64. Rees BB, Hand SC. Biochemical correlates of estivation tolerance in the mountainsnail Oreohelix (Pulmonata: Oreohelicidae). Biol Bull. 1993;184(2):230-242.

65. Chalifour B, Hoogveld JR, Derksen-Hooijberg M, Harris KL, Urueña JM, Sawyer WG, Angelini C. Drought alters the spatial distribution, grazing patterns, and radula morphology of a fungal-farming salt marsh snail. Mar Ecol Prog Ser. 2019;620:1-13.

66. Glasheen PM, Calvo C, Meerhoff M, Hayes KA, Burks RL. Survival, recovery, and reproduction of apple snails (Pomacea spp.) following exposure to drought conditions. Freshw Sci. 2017;36(2):316-324.

67. Van Horn DJ, Garcia JR, Loker ES, Mitchell KR, Mkoji GM, Adema CM, et al. Complex intestinal bacterial communities in three species of planorbid snails. J Molluscan Stud. 2012;78(1):74-80.

68. O'Rorke R, Cobian GM, Holland BS, Price MR, Costello V, Amend AS. Dining local: The microbial diet of a snail that grazes microbial communities is geographically structured. Environ Microbiol. 2015:17(5):1753-64.

69. Charrier M, Combet-Blanc Y, Ollivier B. Bacterial flora in the gut of Helix aspersa (Gastropoda Pulmonata): evidence for a permanent population with a dominant homolactic intestinal bacterium, Enterococcus casseliflavus. Can J Microbiol. 1998;44(1):20-27.

70. Charrier M, Fonty G, Gaillard-Martinie B, Ainouche K, Andant G. Isolation and characterization of cultivable fermentative bacteria from the intestine of two edible snails, Helix pomatia and Cornu aspersum (Gastropoda: Pulmonata). Biol Res. 2006;39:669-681.

71. Niu X, Song L, Xiao Y, Ge W. Drought-tolerant plant growth-promoting rhizobacteria associated with foxtail millet in a semi-arid agroecosystem and their potential in alleviating drought stress. Front Microbiol. 2018;8:2580. 
72. Guo DJ, Singh RK, Singh P, Li DP, Sharma A, Xing YX, et al. Complete genome sequence of Enterobacter roggenkampii ED5, a nitrogen fixing plant growth promoting endophytic bacterium with biocontrol and stress tolerance properties, isolated from sugarcane root. Front Microbiol. 2020;11:2270.

73. Lesser MP, Fiore C, Slattery M, Zaneveld J. Climate change stressors destabilize the microbiome of the Caribbean barrel sponge, Xestospongia muta. J Exp Mar Biol Ecol. 2016;475:11-18.

74. Reese AT, Dunn RR. Drivers of microbiome biodiversity: a review of general rules, feces, and ignorance. MBio. 2018;9(4):e01294-18.

75. Benson AK, Kelly SA, Legge R, Ma F, Low SJ, Kim J, et al. Individuality in gut microbiota composition is a complex polygenic trait shaped by multiple environmental and host genetic factors. PNAS. 2010;107(44):18933-18938.

76. Mandrioli M. Insect collections and DNA analyses: how to manage collections? MMC. 2008;23(2):193-199.

77. Cooper A. DNA from Museum Specimens. In: Herrmann B, Hummel S, editors. Ancient DNA: Recovery and Analysis of Genetic Material from Paleontological, Archaeological, Museum, Medical, and Forensic Specimens. 1994. p. 149-165.

\section{Figures}

\section{Figure 1}

(A) Two adult Oreohelix strigosa individuals (circled) grazing in leaf litter, (B) a dry O. strigosa specimen from the University of Colorado Museum of Natural History (University of Colorado Museum (UCM) Number 2928, originally collected from Morrison, Colorado, USA, in September 1911), and (C) a dissected and complete $O$. strigosa internal body with arrow pointing to where gut tissue was extracted, alongside shell (adapted from Figure 1 in [20]). Photo A by J. Li, photos B and C by B. Chalifour. 


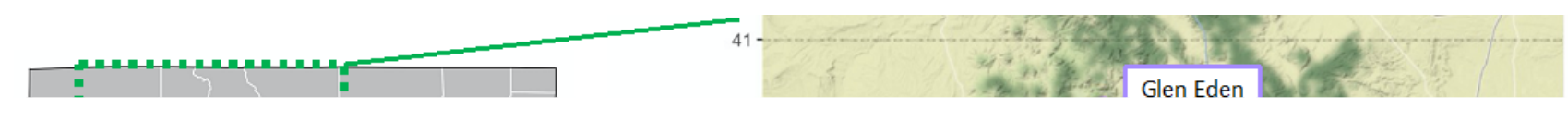

\section{Figure 2}

Map of the Front Range region of the state of Colorado, with collection points and their corresponding years indicated.

\section{Figure 3}

(A) Relative abundance of the top ten bacterial families contributing to each snail sample set collected from the years 1920-2018 and (B) relative abundance of the top ten bacterial families contributing to each snail sample set from 2018. "Other" refers to the cumulative abundance of all other families not included in the top ten.

\section{Figure 4}

Non-metric multidimensional scaling analysis based on (A) year collected, for all snails from 1920-2018 (PERMANOVA: $p$-value $<0.001, \mathrm{R}^{2}=0.02$ ), (B) location collected, for all snails from 1920-2018 (PERMANOVA: $p$-value $<0.001, \mathrm{R}^{2}=0.12$ ), (C) short term preservation treatment, for all snails from 2018 
(PERMANOVA: $p$-value $<0.001, R^{2}=0.20$ ), and $(D)$ location collected, for all snails from 2018 (PERMANOVA: $p$-value $<0.001, R^{2}=0.18$ ). Each point represents one snail gut microbiome community. MRS refers to the Mountain Research Station location.

\section{Figure 5}

Average microbial richness in varying short-term preservation treatments, differentially across locations. Treatments that underwent no preservation prior to DNA extraction (Fresh and Starved) had significantly higher richness. $\left(^{*}\right)$ indicates significant difference. Error bars indicate standard error.

\section{Supplementary Files}

This is a list of supplementary files associated with this preprint. Click to download.

- ChalifourElderLiSupplementaryTable1.xlsx 